\section{La macroestructura semántica como estrategia didáctica en la comprensión de textos expositivos}

\author{
María Helena González García* \\ Nora Yanet Zambrano Villamil **: \\ Carlos Alberto Molina Rodríguez $* * * *$
}

Artículo de Reflexión

Fecha de Recepción: 15 septiembre 2017.

Fecha de Aprobación: 3 febrero 2018.

A pesar de que leer es la base de casi todas las actividades que se llevan a cabo en la escuela, y de que la concepción de la lectura como acto comprensivo es aceptada por todo el mundo, la mayoría de investigaciones sobre las actividades de lectura en la escuela demuestran que en ellas no se enseña a entender los textos.

Teresa Colomer

\section{Resumen}

En la presente ponencia se dan a conocer los avances de la investigación que se lleva a cabo con estudiantes de grado noveno en la I. E. José María Córdoba, ubicada en la vereda Merchán, Saboyá, Boyacá. El proyecto pretende mostrar, mediante el uso de los organizadores gráficos como estrategia didáctica, el impacto del reconocimiento de la macroestructura semántica (M S) en la comprensión lectora de textos expositivos. Al momento de la lectura, la M S, como una forma de acercamiento al texto para identificar tanto el tema general como los específicos, permite la comprensión y la aprehensión de un texto. Como antecedentes, se citan, algunos trabajos que han abordado, desde diferentes perspectivas el tema: cuatro internacionales y dos nacionales. Metodológicamente, la
* Institución Educativa José María Córdoba - Boyacá Colombia mahegoga10@gmail.com **Institución Educativa José María Córdoba - Boyacá Colombia

nyzamvilla@hotmail.com *** Universidad Pedagógica y Tecnológica de Colombia - Boyacá - Colombia carlos.molina@uptc.edu.co

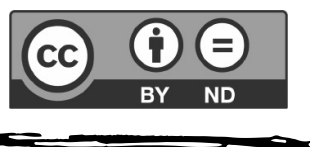


propuesta se desarrolla a partir de la investigación acción en el aula con enfoque mixto cualitativo y cuantitativo, con mayor énfasis en el enfoque cualitativo; se fundamenta en guías didácticas alrededor de algunos organizadores gráficos como elementos esenciales para hacer evidente la macroestructura. La ponencia es el avance de una investigación en proceso y los resultados se analizarán posteriormente para evaluar el impacto en la comprensión lectora, según las pruebas internas y externas de la Institución.

Palabras clave: Semántica, estrategia de enseñanza, comprensión, lectura, investigación. 


\section{Introducción}

La Institución Educativa José María Córdoba, fundada el 20 de enero 1981, se encuentra ubicada en la vereda de Merchán, a $11 \mathrm{~km}$ de la zona urbana del municipio de Saboyá Boyacá, zona de clima frio de páramo y cuya temperatura oscila entre los $6^{\circ}$ y los $14^{\circ}$ centígrados.

En concordancia con los resultados obtenidos por los estudiantes en las pruebas Saber, se puede reconocer que, en el transcurso de los tres últimos años, la institución ha obtenido resultados bajos en relación con el municipio, el departamento y la nación respecto a las pruebas de grado 9 . La aplicación de las pruebas Saber tiene como propósito el mejoramiento de la calidad de la educación a través de la evaluación de competencias básicas, transversales a las áreas curriculares del conocimiento, relacionadas con los estándares de calidad y derechos básicos de aprendizaje que establece el Ministerio de Educación Nacional para identificar fortalezas, debilidades y proponer planes de mejoramiento.

Como factores externos que pueden incidir de manera negativa en el nivel académico de los estudiantes de la institución se pueden señalar: aspectos familiares (bajos ingresos económicos, arduo trabajo agrícola, poco apoyo de padres a hijos, violencia intrafamiliar, falta de afecto, desintegración familiar), aspectos personales (desnutrición, falta de expectativas para un proyecto de vida adecuado que propenda en un mejoramiento profesional y laboral), aspectos escolares (bajo nivel de comprensión lectora, desinterés hacia el aprendizaje, difícil acceso a las tics e internet, ausencia de una biblioteca Institucional).

Los estudiantes de la I. E. José María Córdoba, de acuerdo con las evaluaciones internas y externas, muestran bajos procesos de comprensión lectora que les facilite mejorar el desempeño lector de textos expositivos y avanzar en prácticas que permitan transformar y generar condiciones adecuadas en su quehacer educativo.

El grado noveno cierra el ciclo de formación básica y abre el camino hacia la educación media; sin embargo, a este grado los estudiantes llegan con un nivel de comprensión mínima: en su mayoría, presentan dificultades a la hora de interpretar un texto sin importar el área del conocimiento que estén desarrollando.

De acuerdo con lo anterior, se pretende mostrar, mediante el uso de algunos organizadores gráficos, el impacto que tiene el reconocimiento de la macroestructura semántica en el mejoramiento de la comprensión lectora de textos expositivos en estudiantes del grado noveno de la I. E. José María Córdoba de la vereda de Merchán, Saboyá.

De otra parte, se debe reconocer el papel de la lectura en la educación; sin importar el área del conocimiento, el docente, en su proceso de enseñanza, siempre está solicitando a sus estudiantes que lean e interpreten textos referidos a los temas que se están desarrollando para, de esta manera,
El grado noveno cierra el ciclo de formación básica y abre el camino hacia la educación media; sin embargo, a este grado los estudiantes llegan con un nivel de comprensión mínima 
Elliot (1993) define la investigación-acción como "un estudio de una situación social con el fin de mejorar la calidad de la acción dentro de la misma" (citado por Rodríguez et al. 2011, p. 4). facilitar la comprensión y el manejo de la situación expuesta. Cuando el estudiante entiende lo que lee en las diferentes disciplinas, requiere unos aprendizajes específicos referentes a la lectura para dar un sentido significativo al texto. Así, se hace importante que los estudiantes, durante el transcurso de su vida escolar, desarrollen estrategias de comprensión lectora que les permitan activar su conocimiento, tanto en el conjunto de acciones integradas de las diferentes asignaturas en pruebas internas, como en la presentación de pruebas externas; de ahí la importancia de contar con habilidades que favorezcan la interpretación textual.

Ahora bien, en el contexto escolar, la enseñanza de las distintas ciencias, en su gran mayoría, se realiza mediante textos expositivos que desarrollan y explican diferentes temáticas. Así, esta clase de escrito se constituye en una herramienta de aprendizaje que permite la adquisición de conocimientos, el desarrollo del componente cognitivo de la persona, la reflexión y la formación de conceptos que propician prácticas formativas. La información referencial ofrecida es trascendental en la adquisición de conocimientos, el manejo de vocabulario y la organización de ideas. Por ello, se hace necesario profundizar en el manejo de textos expositivos a través de estrategias didácticas que se desarrollan a partir de la macroestructura semántica.

Desde el punto de vista metodológico, el proyecto propone adelantar una investigación-acción, ante la necesidad de generar cambios que se dirijan hacia el aprovechamiento de las potencialidades del estudiante de manera innovadora, dinámica y participativa, a manera de exploración de instrumentos o de herramientas de aprendizaje, se plantea la necesidad de tener en cuenta no solo la teoría, sino también la práctica. Así, la investigación-acción se constituye en un método de carácter colectivo que busca convertir en eje central lo que ocurre en la práctica educativa, con el fin de identificar aspectos que puedan ser mejorados mediante estrategias sometidas a la observación, reflexión y cambio; lo anterior en pro de una transformación social desde el punto de vista educativo en el lugar donde se desarrolla.

Elliot (1993) define la investigaciónacción como "un estudio de una situación social con el fin de mejorar la calidad de la acción dentro de la misma" (citado por Rodríguez et al. 2011, p. 4). Entonces, la investigación acción y su implementación en las aulas repercute en el mejoramiento de los resultados y, por ende, en la calidad educativa. Además, permite una autorreflexión del quehacer educativo tanto de los profesores como de los estudiantes y demás personas que intervienen en el proceso.

La investigación-acción tiene como particularidad la repetición de pasos similares (cíclica), sigue un orden en el proceso,(planificar, actuar, observar y reflexionar); implica una participación activa de todos los involucrados en la investigación, es reflexiva crítica sobre el proceso y los resultados de manera que va cambiando a medida 
que se va realizando, es visible y abierta a sugerencias de otros que de una u otra manera se comprometen $y$ con el adecuado consentimiento empiezan a hacer parte del proceso de investigación.

El enfoque de la investigación es mixto: cualitativo y cuantitativo con mayor énfasis en el enfoque cualitativo. Se acude al enfoque cuantitativo porque hay una conducta de entrada que arroja unas estadísticas respecto de la comprensión lectora de textos expositivos de los estudiantes de la muestra y una conducta de salida que proporciona unas estadísticas después de aplicadas las estrategias con respecto a los resultados finales. El enfoque cualitativo está centrado en la investigación-acción que trabaja con grupos en el aula mediante la implementación de guías didácticas, portafolios y organizadores gráficos desde donde se maneja la macroestructura semántica como estrategia didáctica para mejorar la comprensión.

La población está constituida por cincuenta estudiantes de grado noveno de la I. E. José María Córdoba del municipio de Saboyá, cuyas edades oscilan entre los catorce y los dieciséis años. El análisis se hará con una muestra de diez estudiantes seleccionados aleatoriamente.

\section{Antecedentes}

Se pueden citar, en principio, seis trabajos que han abordado, desde diferentes perspectivas, el tema de la macroestructura semántica como estrategia didáctica para la comprensión de textos académicos. Cuatro a nivel internacional trabajos y dos en el ámbito nacional sirvieron como referencia para nutrir esta investigación.

Comprender y representar un texto en la memoria. Investigación realizada por José Otero Gutiérrez en Alcalá de Henares, España. Con base en teorías de Van Dijk y de Kintsch, Otero pretende como objetivo general analizar la microestructura, la macroestructura y la superestructura en la comprensión de un texto. Esboza el uso de tres niveles básicos de simbolización memorística: formulación superficial (representación literal), base de texto (tópico de texto) que comprende la micro, la macro y la superestructura, y el modelo de la situación (representación cognitiva de generalidades sobre el texto). Otero concluye que tanto autor como lector deben estar estrechamente ligados, tener en cuenta el modelo de la situación y el manejo de los conocimientos previos para de esta manera relacionar la información que proporciona el autor con la que el lector tiene.

Construcción de la microestructura y la macroestructura semántica en textos expositivos producidos por estudiantes universitarios de LUZ. Investigación adelantada en Venezuela por Gloria Fuenmayor, Yeriling Villasmil, María Rincón (Facultad de Humanidades y Educación, Universidad del Zulia). Aquí se analiza la construcción de la micro y la macroestructura semántica en textos expositivos producidos por estudiantes 
universitarios, a partir del uso de procedimientos gramaticales como las proformas. Con base en teorías de Van Dijk, de Kintsch y de Álvarez, se reconoce que los estudiantes de la muestra elaboran textos expositivos manteniendo un tema pero fallan en la producción de la microestructura textual porque desconocen los mecanismos de coherencia y cohesión textual; de igual manera, producen textos expositivos pero sin una macroestructura apropiada.

Propuesta de lectura desde la lingüística textual. Investigación desarrollada por Hugo González Aguilar en Perú, cuyo objetivo consiste en comprender un escrito a partir de la lingüística textual. Tiene en cuenta diversos autores como Todorov, Greimas, Vygotsky, Ausubel, Bruner y Van Dijk. Se trata de una propuesta de lectura fundamentada en planteamientos sobre superestructura, macroestructura (supresión, generalización y construcción), microestructura, componente crítico y creativo y esquematización de la información. Concluye que, mediante el uso de la lingüística textual, se puede interpretar textos literarios haciendo uso de organizadores gráficos y de estructura de una narración.

El nivel atencional en la comprensión textual. Investigación adelantada por la argentina Verónica Carmen Mendizábal que tiene como objetivos describir y analizar las capacidades de comprensión del lector. En el marco teórico hace referencia a aportes de investigadores sobre la adquisición de conocimientos relacionados con la lectura. El proyecto se fundamenta en el modelo de Van Dijk, sobre esquemas conceptuales de super y macroestructura los que se debe tener en cuenta en una tipología textual. Concluye que es tarea del profesor facilitar el tiempo y los momentos para que el estudiante haga práctica de la lectura desde su propio interés.

Guía para el fortalecimiento en la comprensión de textos tipo pruebas saber. Investigación desarrollada por Marcela Álzate Arbeláez, realizada con estudiantes de grado noveno de la Institución Educativa Santa Sofía ubicada en Dos Quebradas, Risaralda. La investigadora formula una propuesta pedagógica basada en la teoría de Teun A. Van Dijk, con la cual busca el mejoramiento en comprensión e interpretación de textos tipo pruebas Saber; además, comprueba que las debilidades se encuentran principalmente en la falta de motivación por la lectura y la falta de implementación en el manejo de test de comprensión lectora desde los grados inferiores.

Incidencia de una secuencia didáctica desde una perspectiva discursivainteractiva en la comprensión lectora de textos expositivos de estudiantes de grado $9^{\circ}$ de EBS, de la I. E. INEM Felipe Pérez de la ciudad de Pereira. Como propósito tiene el presentar los resultados de la implementación de una estrategia de enseñanzaaprendizaje enmarcada en la didáctica de la lengua, desde una perspectiva teórica discursivo-interactiva en busca 
de mejorar procesos de comprensión de textos expositivos con estudiantes de grado noveno. Durante el trabajo se hicieron explícitas las formas de organización superestructura de este tipo de texto.

\section{La macroestructura semántica.}

La lingüística como ciencia desarrolla diferentes niveles de estudio: fonéticofonológico, morfo-sintáctico, semántico y pragmático. En el nivel semántico, cuyo propósito es el análisis del significado y el sentido, se puede ubicar el concepto de macroestructura semántica (referido, en principio, al tema o tópico de un texto). Dicho concepto fue formulado por el lingüista holandés Teun A. Van Dijk hacia 1977. De acuerdo con Van Dijk, un texto se organiza en dos estructuras principales que dan cuenta de su contenido: la súper estructura y la macro estructura. Un escrito que no atienda a estas dos estructuras no puede ser considerado como tal porque no tiene coherencia global (Van Dijk, 1996, p. 53). El mismo autor explica: "las macroestructuras semánticas son la reconstrucción teórica de nociones, tema o asunto del discurso" (1996, p. 43).

El texto, sin importar la clase a la que corresponda, atiende a un todo de significado y de sentido: un tema. Ahora bien, el sentido global de un texto se logra por la relación de sus partes; es decir, por la conexión de sus oraciones acorde a una coherencia lineal $y$, a la vez, de sus partes que vienen a ser los párrafos. Aquí surgen las proposiciones que son oraciones gramaticalmente completas con las cuales se expresa un pensamiento, describen un determinado estado de cosas y hacen parte de la macro estructura. Al respecto, Van Dijk dice: "las proposiciones no solo se conectan linealmente la una de la otra, sino que una secuencia de ellas debe estar relacionada de manera más global, por medio de un tema común” (1980, p. 47). También asegura: "las proposiciones tienen la capacidad especifica de ser verdaderas o falsas, o de permitir que las oraciones que las expresan se llamen verdaderas o falsas. A grandes rasgos, una proposición es el significado que subyace en una clausula $\mathrm{u}$ oración simple” (Van Dijk, 1996, p. 27).

El deducir un tema o tópico textual requiere diversas estrategias; entre ellas, las macrorreglas que consisten en los pasos para llegar a la macroestructura semántica.

Supresión. Dada una secuencia de proposiciones, se suprimen todas las que no sean presuposiciones de las proposiciones subsiguientes de la secuencia.

Generalización. Dada una secuencia de proposiciones, se hace una proposición que contenga un concepto derivado de los conceptos de la secuencia de proposiciones, y la proposición así construida sustituye a la secuencia original.

Construcción. Dada una secuencia de proposiciones se bace una proposición que denote el mismo becho denotado por la totalidad de la secuencia de
Van Dijk dice: "las proposiciones no solo se conectan linealmente la una de la otra, sino que una secuencia de ellas debe estar relacionada de manera más global, por medio de un tema común" 
proposiciones, y se sustituye la secuencia original por la nueva proposición (Van Dijk, 1980, p. 48).

En la comprensión textual se hace necesario saber el tipo de texto y su estructura; esto es, la superestructura semántica: "una superestructura puede caracterizarse intuitivamente como la forma global de un discurso, que define la ordenación global del discurso y las relaciones (jerárquicas) de sus respectivos fragmentos" (Van Dijk, 1980, p. 53).

Deotrolado, alhablardemacroestructura se está haciendo referencia a la identificación de la estructura profunda y superficial de un texto. Bernal reconoce un macrocomponente $\mathrm{y}$ un microcomponente textual. El primero se relaciona con la intención comunicativa del autor; el segundo, la estructura superficial del texto; la macroestructura reúne los anteriores aspectos (1984, p. 267). Asimismo, habla de la macroestructura semántica como: "el desarrollo transformativo, jerarquizado y coherente, de un tópico inicial o tópico del discurso. Dicho tópico estaría constituido de manera que contuviera en si toda la información esencial del texto desarrollado" (Bernal, 1984, p. 267).

\section{El texto expositivo.}

De acuerdo con la tipología textual de Werlich (1976), los textos expositivos se producen en todas las ciencias, tanto en las físico-matemáticas, biológicas como en las sociales, dado que el objetivo de las ciencias consiste en proporcionar explicaciones a los fenómenos característicos de cada uno de sus campos (citado por Martínez y Rodríguez, 1989 p.78).

Al texto expositivo como herramienta esencial en el aprendizaje $y$ en la enseñanza no se le ha dado la importancia que tiene. En la enseñanza de la lengua se dedica más tiempo al trabajo de textos narrativos, descriptivo o liricos y se deja de lado la información referencial que brinda este tipo de discurso. Al respecto, Martínez y Rodríguez dicen: "el texto expositivo resulta ser el texto escolar por excelencia; tanto en el discurso del profesor cuando 'explica' como en el de los alumnos, ya sea para transmitir las informaciones o para elaborar nuevas informaciones requeridas por el trabajo escolar” (1989, p. 78).

Todas las áreas del saber fundamentan su enseñanza en el uso del texto expositivo; convirtiéndose en una ayuda interdisciplinar que a través del uso de la lengua forman esquemas mentales en la adquisición e incremento del conocimiento. Martínez y Rodríguez lo sustentan de la siguiente manera: "el texto expositivo supone un marco de trabajo privilegiado para la interdisciplinariedad y el trabajo significativo de las estrategias lingüísticas y comunicativas en general" (1989, p. 79)

Definición. El texto expositivo consiste en un escrito que desarrolla unos conceptos mediante la explicación de un tema en forma clara y objetiva, aclarando, informando $\mathrm{y}$, a la vez, ampliando el conocimiento de un determinado receptor o lector. Frías 
define al texto expositivo como: "aquel que cumple una función referencial. $\mathrm{Su}$ principal objetivo es informar, incluyendo comentarios aclaratorios, incorporando explicaciones y utilizando claves explicativas (título, sub-títulos, alusiones)" (2008, p. 115). A su vez, Sánchez asegura que "los textos expositivos presentan el resultado de un estudio, una reflexión, una investigación o un trabajo sobre un asunto o tema para darlo a conocer y explicarlo" (2009, p. 68).

Características. De acuerdo con el propósito comunicativo, el texto expositivo cumple con las siguientes características: conceptualiza conocimientos, se estructura en introducción, desarrollo y conclusión, desarrolla un tema y pueden tener subtemas, informa y amplía el conocimiento por medio de la explicación.

Agosto y Picó (2013) indican que la superestructura de un texto expositivo está conformada por tres partes: "Introducción, que trata de contestar a las preguntas ¿qué?, ¿por qué? y ¿cómo?, el desarrollo, etapa en la que se presenta la resolución por medio de la explicación del tema (...) y, en última instancia, la conclusión con la que se cierra la exposición de la información" (citados por Oliveros, 2013, p. 24).

Organización. El texto expositivo, a diferencia de otros tipos de texto, no presenta una estructura igual en el desarrollo del tema. Álvarez y Ramírez (2013) proponen la siguiente clasificación: a) Definición/descripción (explica el tema según la secuencia: qué es, cuáles son sus características).

b) Clasificación/tipología (desarrolla tipos o clases a través de la enumeración de los rasgos)

c) Comparación/contraste (subraya las diferencias y similitudes de varios fenómenos o realidades).

d) Expositivo de problema-solución (expone diversas formas para resolver una problemática).

e) Expositivo de pregunta-respuesta (expresa la necesidad de saber y luego explica el contenido).

f) Expositivo de causa-consecuencia (explica las consecuencias que causa un fenómeno).

g) Expositivo de ilustración (expone en forma de planos, gráficos, tablas, esquemas, etc.). (Citados por Oliveros 2013, pp. 27-28).

Estas formas de clasificación tienen como finalidad primordial ilustrar un tema y exponerlo para ampliar la información acorde a la intención comunicativa del autor del texto.

\section{La lectura compresiva y su importancia.}

Este tipo de lectura se puede definir como el acto de entrar en diálogo con el texto, apropiarse del mismo, interpretar el mensaje del autor y, de esta manera, asumir una posición frente a la información presentada; permite ampliar el conocimiento y construir 
significados que simultáneamente se van asociando al contexto o situación en la que se encuentre determinado lector. De acuerdo con Cassany, la persona que tiene lectura comprensiva se destaca porque:

Comprende autónomamente el propósito lingüístico, las intenciones pragmáticas y los puntos de vista particulares que subyacen a los discursos que le rodean;

Toma conciencia del contexto (contenido cognitivo, genero discursivo, comunidad de bablantes, etc.) desde el que se han elaborado dichos discursos;

Utiliza todos los recursos lingüísticos disponibles para conseguir interpretar el texto leído (2003, p. 114).

La lectura compresiva puede definirse también como una actividad constructiva, interactiva y estratégica. Constructiva porque el lector no traspasa a su memoria lo expuesto por el texto; por el contrario, se apropia de la información con el fin de construir conocimiento. Interactiva porque la interpretación y la construcción se realizan mediante la interacción activa entre el lector, el texto y el contexto. Estratégica porque un buen lector usa estrategias de lectura con el fin de cumplir con sus objetivos y su plan, aunque estos sean implícitos (Liceo Javier, 2013, p. 13).

En el momento de iniciar una lectura en la que el propósito es aprender y adquirir nuevos conocimientos, el lector activa sus saberes previos para conectarlos con la nueva información y luego hacer una selección lo que le es útil para construir un conjunto de conocimientos producto del nuevo aprendizaje. Solé (1998) al respecto afirma: “... nos vemos obligados a efectuar una revisión de dicho conocimiento para que pueda integrarse la nueva y/o contradictoria información. Esta información puede tener múltiples resultados: ampliación del conocimiento previo con la introducción de nuevas variables, modificación radical de este, establecimiento de relaciones nuevas con otros conceptos...” (p. 38).

Cuando se realiza una verdadera lectura comprensiva, se pueden evidenciar los resultados de dicha información en la capacidad que demuestra el lector al encontrarse inmerso dentro de un cúmulo de conocimientos que le aportan estructuras cognitivas en las que se modifican y amplían conductas. "cuando un lector comprende lo que lee, está aprendiendo en la medida en la que su lectura le informa, le permite acercarse al mundo de significados de un autor y le ofrece nuevas perspectivas $\mathrm{u}$ opiniones sobre determinados aspectos, etc. La lectura nos acerca a la cultura (...) a múltiples culturas y, en ese sentido, siempre es una contribución esencial a la cultura propia del lector". (p. 39).

\section{Metacognición.}

Burón (1993) define la metacognición como: "el conocimiento de las distintas operaciones mentales y saber cómo, cuándo y para qué se deben usar. Más tarde, amplía el concepto y lo define como el conocimiento y regulación 
de las propias cogniciones y de los propios procesos mentales: percepción, atención, memorización, lectura, escritura, comprensión, comunicación: qué son, cómo se realizan, cuándo hay que usar una u otra, qué factores ayudan o interfieren su operatividad. Quizá sería mejor llamarla conocimiento autorreflexivo, "puesto que se refiere al conocimiento de la propia mente adquirido por autoobservación" (citado por Jiménez 2004, p. 47).

Otros autores, como Ormrod (2005), definen la metacognición como "el conocimiento que tiene el individuo de su propio proceso cognitivo y de aprendizaje, así la capacitad para regular el mismo" (citado por Tapia, Terrero y Cabral, 2015, p.2)

En el caso de la lectura, el individuo regula su proceso de comprensión mediante la aplicación de estrategias metacognitivas que le ayudarán a dosificar su aprendizaje. El lector organiza un plan que adecua acorde a su propósito y al tipo de texto, es un autodidacta que estructura la información haciendo inferencia, identificando palabras o conceptos claves y elaborando resúmenes. Baker y Brown (1984a) "distinguen entre dos tipos de lectura: leer para comprender, que implica la supervisión de la comprensión; y leer para aprender (algo más que leer para comprender), que implica la identificación de las ideas principales seleccionando lo más relevante del texto, el análisis de las demandas de los materiales a utilizar, el uso y mantenimiento de estrategias adecuadas evaluando su efectividad y el establecimiento de un horario y un clima adecuado para el estudio" (citados por Jiménez, 2004, p. 83)

Cañón, Mancera y Ruiz (2010) dicen: "si lo cognitivo se relaciona con el conocimiento, lo metacognitivo se refiere al hecho de tener conciencia de tal conocimiento y de cómo se logra". (p. 127). La metacognición cumple una función importante en el modelo pedagógico de Escuela Activa puesto que el estudiante es capaz de evaluar el nivel de su propio aprendizaje en situaciones de autorreflexión. Tanto el docente como el estudiante están inmersos en el proceso educativo haciendo uso de diferentes tipos de evaluación de manera que, luego de un análisis y valoración de resultados, el estudiante es capaz de emitir unos juicios valorativos.

\section{Organizadores gráficos.}

Son herramientas que facilitan la comprensión textual puesto que después de leer un escrito, la información se puede compilar a través de un organizador gráfico. Este tipo de esquemas se involucran dentro del aprendizaje visual para ayudar a organizar la información de una manera coherente y aprehensible de tal forma que el conocimiento sea jerarquizado y ayude al estudiante en la construcción de esquemas mentales en el proceso de enseñanza aprendizaje, relacionándolos con su saber previo. Los organizadores gráficos se relacionan con la macroestructura semántica porque representan mental y visualmente de lo que trata el
Cañón, Mancera y Ruiz (2010) dicen: "si lo cognitivo se relaciona con el conocimiento, lo metacognitivo se refiere al hecho de tener conciencia de tal conocimiento y de cómo se logra”. 
La macroestructura semántica representada en cada organizador gráfico conduce a la interpretación global del texto y a la aprehensión del mismo. texto; organizan la información de tal manera que el lector después de realizar el organizador gráfico de determinada lectura, puede con sus propias palabras decir de lo que habla el texto y en efecto mostrar un nivel de comprensión y una visión global del tema. La macroestructura semántica representada en cada organizador gráfico conduce a la interpretación global del texto y a la aprehensión del mismo.

La presente investigación trabaja el texto expositivo a partir de los siguientes organizadores gráficos

Mapa semántico. Descrito inicialmente por Pearson y Johnson en 1978. Se define como una representación visual de un tema que mediante una lluvia de ideas asocia conceptos, palabras claves e ideas que giran alrededor de un tópico general. No hace uso de conectores, utiliza figuras geométricas que van relacionadas por medio de líneas y llama la atención por el manejo del color de acuerdo con la comprensión y la creatividad de su autor; es una técnica que facilita la comprensión textual del estudiante.

Cuadro sinóptico. Resumen esquematizado de una lectura que permite jerarquizar la información tanto de forma horizontal como vertical, se pueden utilizar llaves que ayudan en la esquematización de la información. Sus componentes son idea general, ideas secundarias y detalles.

Resumen en esquemas. Organizan la información de un texto por medio del análisis de la idea principal de cada párrafo con sus respectivos detalles. Aquí se retoma la división lógica que tienen los nomencladores, el uso de palabras e ideas clave. Ha sido trabajado por la docente Ligia Cañón Flórez en una investigación de comprensión lectora.

Esquema causa efecto o espina de pescado. Utilizado por primera vez en 1953 por el profesor Karou Ishikawa. Se utiliza para visualizar las causas que originan una consecuencia o problema. Está conformado por un recuadro al que se le denomina cabeza y una línea principal llamada columna vertebral; de esta línea se derivan otras que desarrollan unas causas y de acuerdo con estas, unas consecuencias.

Mapa conceptual. Se empezó a utilizar en 1960 por el profesor Joseph Donald Novak. Es la representación gráfica de un conocimiento conformada por una red de conceptos relacionados por palabras conectoras; se caracteriza por la jerarquización de la información; es decir, se organiza en orden de importancia; selección o síntesis significativa e impacto visual, haciendo uso del color y relacionando ideas en orden de importancia.

\section{Estrategias didácticas.}

En el contexto educativo estrategia es el diseño, planeación y ejecución de actividades pedagógicas, criticoreflexivas por parte de los docentes para conseguir o lograr una meta y conllevan a la profundización y creación de conocimientos que enriquezcan la conciencia colectiva de los estudiantes. 
El trabajo con material didáctico, actividades agradables y novedosas para los estudiantes en la búsqueda de su formación, son aporte importante en pro del mejoramiento respecto de los avances en la práctica educativa. Rodríguez (2007) define estrategias como: "en el campo de la pedagogía, las estrategias didácticas se refieren a tareas y actividades que pone en marcha el docente de forma sistemática para lograr unos determinados objetivos de aprendizaje" (p.3).

En este proyecto de investigación se usa como estrategia didáctica el manejo de algunos organizadores gráficos que sirven como herramienta para identificar el tópico de textos expositivos y llegar a una comprensión lectora mediante información que evita la memorización pero sí el análisis textual, especificando la información a la que se hace referencia.

\section{Resultados y discusiones.}

El desarrollo de este proyecto inició con la lectura y el análisis de documentos institucionales para conocer cómo, desde el PEI y el ISCE, se puede verificar resultados de pruebas externas (SABER) para identificar el problema y proponer una alternativa de solución; a continuación se realizó una encuesta de cinco (5) preguntas cuyo propósito fundamental radica en identificar conocimientos previos respecto de: Macroestructura semántica, texto expositivo, organizadores gráficos y comprensión lectora. Esta encuesta se aplicó a los 50 estudiantes del grado noveno de la I. E. José María Córdoba, quienes se encuentran distribuidos en dos grupos. Estas fueron las preguntas:

1. ¿Identifica las características propias de un texto expositivo?

2. Cuándo lee un texto, ¿Identifica la idea principal?

3. ¿Maneja conocimientos respecto a lo que son los organizadores gráficos?

4. ¿Infiere la idea principal de un párrafo?

5. Frente a un texto, ¿es capaz de organizar la información en su orden de importancia?

En cada pregunta se presentaron cinco opciones de respuesta (Siempre, Casi siempre, Algunas veces, Casi nunca, Nunca).

En las figuras 1, 2, 3, 4 y 5 se presenta el porcentaje y el análisis de cada una de las respuestas por parte de los estudiantes en la encuesta realizada.

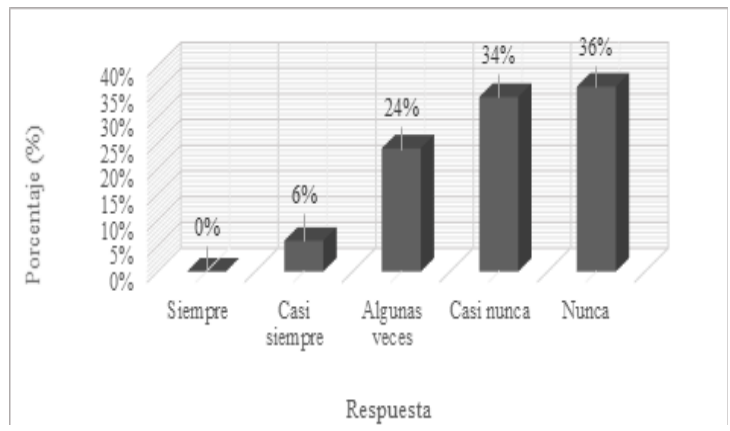

Figura 1. Porcentaje de respuestas para la pregunta número 1.

En relación con la primera pregunta, de la anterior figura, se puede deducir que la mayor parte de la 
población encuestada no identifica las características propias de un texto expositivo; se puede apreciar que el $36 \%$ de los estudiantes nunca y el $34 \%$ casi nunca identifican y diferencian los tipos de texto; frente al 6\% que casi siempre poseen habilidades para caracterizar esta clase de escrito.

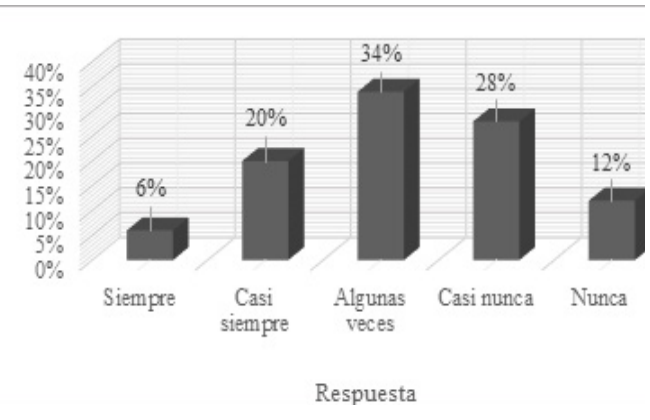

Fuente: Elaboración propia

Figura 2. Porcentaje de respuestas para la pregunta número 2 .

En la segunda pregunta se puede reconocer que el $12 \%$ de los estudiantes nunca y el $28 \%$ casi nunca identifican la idea principal en un texto, frente al $6 \%$ que si deducen el tópico del texto.

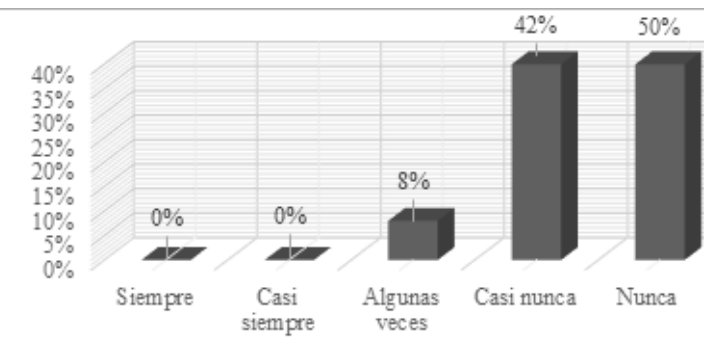

Fuente: Elaboración propia

Figura 3. Porcentaje de respuestas para la pregunta número 3 .
Las respuestas a la pregunta número tres permiten evidenciar que un $92 \%$ de los estudiantes nunca o casi nunca han elaborado organizadores gráficos como estrategia para identificar tópico $y$ aspectos relevantes de un texto; solo el $8 \%$ son capaces de reconocer algunos.

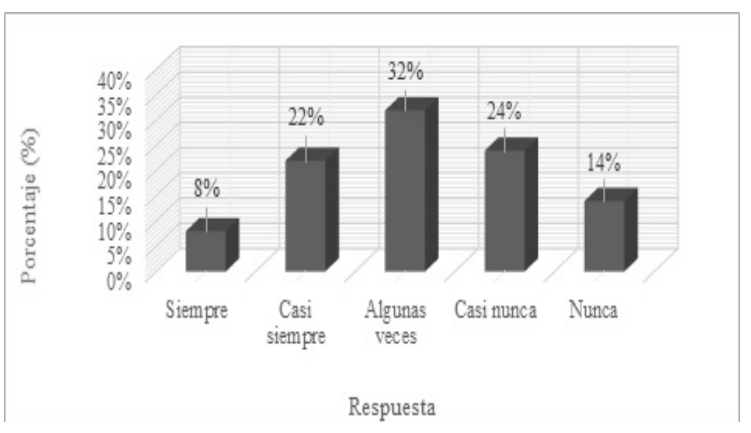

Fuente: Elaboración propia

Figura 4. Porcentaje de respuestas para la pregunta número 4 .

La pregunta número 4 , acerca de la inferencia de la idea principal de un párrafo, se puede observar que los resultados se encuentran equilibrados respecto a lo positivo y negativo: entre casi nunca y nunca, existe un rango de $38 \%$ frente al 30\% del siempre y casi siempre y al $32 \%$ de algunas veces.

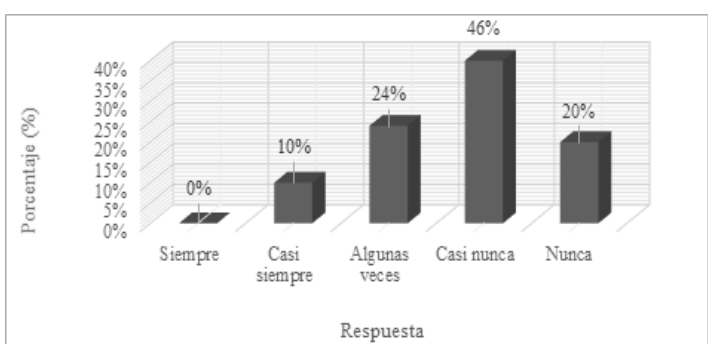

Fuente: Elaboración propia

Figura 5. Porcentaje de respuestas para la pregunta número 5 . 
En la pregunta 5 se observa que los estudiantes presentan dificultades para organizar la información jerárquicamente en un texto puesto que el 66\% casi nunca y nunca son capaces de esquematizar el texto en su orden de importancia, frente a un $10 \%$ que casi siempre lo hacen.

Posteriormente, se llevó a cabo una prueba diagnóstica de lectura. Los estudiantes de los dos grupos de noveno debían elaborar un mapa conceptual a partir de un texto expositivo de la asignatura de Ciencias Naturales, denominado "Gorilas de la Llanura: grandes desconocidos" tomado de la Revista Natura. Se eligió este organizador gráfico puesto que los estudiantes manifestaron tener conocimientos previos y la utilización del mismo en la práctica de aula de las diferentes asignaturas. Después de una observación general se puede deducir que los estudiantes respecto al manejo del mapa conceptual presentan diversas dificultades de acuerdo con las características propias que debe reunir esta clase de organizador:

Se observa que el orden jerárquico en las ideas no es tenido en cuenta, los conceptos son transcritos del texto, en ocasiones con una gran extensión sin tener en cuenta el uso de palabras e ideas claves. Los conectores utilizados no son adecuados puesto que hacen uso de oraciones que se alejan del concepto y la función de estas palabras de enlace. Algunos estudiantes confunden el Organizador grafico Mapa Conceptual con el Cuadro sinóptico. En el momento de la sustentación los estudiantes no dan cuenta de lo que dice el texto y se limitan a leer los párrafos que copiaron del escrito original. Además, no se maneja el color, aspecto importante en la representación visual para una adecuada comprensión.

Finalmente, se puede afirmar: a pesar de que este organizador gráfico es utilizado en la práctica de aula de otras áreas, no está completamente fundamentado con la teoría ni con su respectiva función.

\section{Resultados de la fase diagnóstica}

Al realizar un análisis de las actividades de tipo diagnóstico se puede deducir que la mayoría de los estudiantes dan a entender que existe cierto grado de desconocimiento respecto de la identificación del tópico, idea general, manejo de estrategias lectoras (organizadores gráficos) y estructura textual en la comprensión lectora y la identificación de la macroestructura semántica de un texto expositivo.

Se evidencia que la gran mayoría de estudiantes presentan dificultades respecto al uso de este tipo de estrategias didácticas viéndose reflejado en la confusión que tienen a la hora de elaborar un mapa conceptual; además no manejan las macrorreglas sugeridas por Van Dijk (1980): supresión, generalización y construcción para hallar la macroestructura semántica.

Se puede ratificar que el nivel de comprensión lectora de un texto expositivo es mínimo en los estudiantes de este grado, al igual que el uso del
Se puede afirmar: a pesar de que este organizador gráfico es utilizado en la práctica de aula de otras áreas, no está completamente fundamentado con la teoría ni con su respectiva función. 
organizador grafico Mapa Conceptual como herramienta de trabajo escolar, a pesar de que ha sido utilizado esporádicamente en otras asignaturas.

\section{Propuesta de aplicación}

\section{Guías didácticas.}

La fase de aplicación de la estrategia planteada en el proyecto se hace efectiva mediante el diseño y aplicación de cinco guías o secuencias didácticas (cada una con dos textos expositivos de diferente asignatura). Esta secuencia contempla los siguientes aspectos: nombre del proyecto, estándares asociados, derechos básicos de aprendizaje, objetivo y actividades.
Cada una de las actividades tendrá en cuenta la siguiente estructura: título, tiempo objetivo, procedimiento que contempla tres momentos: uno inicial en el que se realiza la motivación y manejo de conocimientos previos; otro de conceptualización inductiva y ejercicio dirigido por las docentes; y finalmente uno de ejercicio evaluativo con su respectiva retroalimentación y recursos. El desarrollo de cada actividad cuenta con un producto elaborado por los estudiantes y la oportuna orientación de las docentes que los acompañan en todo el desarrollo de la investigación.

Tabla 1

Organizadores gráficos para el desarrollo de la propuesta

\begin{tabular}{|c|c|}
\hline Actividad & Finalidad \\
\hline Mapa semántico & $\begin{array}{l}\text { Organizar las ideas y establecer lazos de relación, integrando las } \\
\text { partes de un todo o desglosando el todo en sus partes. }\end{array}$ \\
\hline Cuadro sinóptico & $\begin{array}{l}\text { Organizar conceptos de lo general a lo particular, y de izquierda } \\
\text { a derecha, en orden jerárquico; haciendo uso de llaves o corchetes. }\end{array}$ \\
\hline $\begin{array}{l}\text { Resumen } \\
\text { esquemas }\end{array}$ & $\begin{array}{l}\text { Comprender la información de un texto expositivo: organizando, } \\
\text { jerarquizando y teniendo en cuenta los niveles de generalidad- } \\
\text { especificidad en relación con el sentido global. }\end{array}$ \\
\hline Espina de pescado & $\begin{array}{l}\text { Identificar todas aquellas relaciones que se presentan entre las } \\
\text { causas y efectos de un proceso dentro de un texto expositivo. }\end{array}$ \\
\hline Mapa conceptual & $\begin{array}{l}\text { Identificar conceptos o ideas clave de un texto, estableciendo } \\
\text { relaciones entre ellos y jerarquizando la información. }\end{array}$ \\
\hline
\end{tabular}

Lo anterior, se trabaja en guías didácticas que estructuran la información teniendo en cuenta los momentos de la clase. 
Tabla 2

Información básica de las guías didácticas

\begin{tabular}{|l|l|}
\hline $\begin{array}{l}\text { I N F O R M A C I Ó N } \\
\text { BÁSICA }^{1}\end{array}$ & $\begin{array}{l}\text { Comprendo e interpreto textos, teniendo en cuenta } \\
\text { el funcionamiento de la lengua en situaciones de } \\
\text { Estándar } \\
\text { interlocutor y del contexto. }\end{array}$ \\
\hline $\begin{array}{l}\text { Derecho básico de de } \\
\text { aprendizaje }\end{array}$ & $\begin{array}{l}\text { Interpreta textos atendiendo al funcionamiento de la } \\
\text { lengua en situaciones de comunicación, a partir del uso de } \\
\text { estrategias de lectura. }\end{array}$ \\
\hline Grado & $9^{\circ}$ \\
\hline Docentes & $\begin{array}{l}\text { María Helena González García } \\
\text { Nora Yanet Zambrano Villamil }\end{array}$ \\
\hline
\end{tabular}

Tabla 3

Estructura general de las guías didácticas

\begin{tabular}{|c|c|}
\hline Número: & \\
\hline \multicolumn{2}{|l|}{ Nombre: } \\
\hline \multicolumn{2}{|l|}{ Fecha: } \\
\hline Tiempo: & horas clase \\
\hline \multicolumn{2}{|l|}{ Objetivo: } \\
\hline $\begin{array}{l}\text { Primera sesión: evocando, estructuro mi pensamiento. } \\
\text { Tiempo: } \\
\text { Objetivo: } \\
\text { Actividades: } \\
\qquad \text { 1. "Reflexión: } \\
\text { 2. "Motivación: } \\
\text { 3. "Manejo de saberes previos: }\end{array}$ & \multirow[t]{3}{*}{ Recursos } \\
\hline $\begin{array}{l}\text { Segunda sesión: Observando aprendo } \\
\text { Tiempo: } \\
\text { Objetivo: } \\
\text { Actividades: } \\
\text { *Conceptualización } \\
\text { *Ejercicio práctico dirigido }\end{array}$ & \\
\hline $\begin{array}{l}\text { Tercera sesión: Practicando organizo información. } \\
\text { Tiempo: } \\
\text { Objetivo: } \\
\text { Actividades: } \\
\text { *Práctica evaluativa } \\
\text { * Socialización, retroalimentación y conclusiones. }\end{array}$ & \\
\hline
\end{tabular}


Los resultados de la presente investigación serán llevados a cabo de modo sistemático y registrado. Se realizará triangulación de los resultados obtenidos, o sea someter a control cruzado para comparar entre sí: diario de campo, guías didácticas y portafolio de los estudiantes. La información obtenida en esta primera triangulación; posteriormente, se confronta con la información arrojada por la indagación cuantitativa, estadística y cualitativa para observar el grado de mejora en los niveles de comprensión de textos expositivos: este es un segundo grado de triangulación de la información.

Con la ejecución de la propuesta descrita anteriormente, se pretende al final del proceso alcanzar un mejoramiento en la comprensión lectora de textos expositivos con estudiantes de grado noveno de educación básica secundaria de la I.E José María Córdoba de Merchán Saboyá.

Con la realización de las guías o secuencias didácticas que usan como herramienta algunos organizadores gráficos y fundamentados en la macroestructura semántica, se tiene la confianza que el desempeño mejore gradualmente tanto en las pruebas internas como externas de acuerdo con la matriz de referencia en lenguaje, emitida por el Ministerio Nacional de Educación Nacional, respecto de la construcción del sentido global del texto y la relación que se establece entre los diferentes campos semánticos para interpretar haciendo uso del resumen, conceptos, palabras e ideas claves.

Se espera que dichas estrategias sean implementadas por docentes de otras áreas en el proceso de enseñanza aprendizaje, teniendo en cuenta la interdisciplinariedad, donde se facilite la comprensión de textos académicos especialmente en las asignaturas de Ciencias Naturales, Sociales y Lenguaje.

\section{Conclusiones}

La lectura comprensiva hace parte del ámbito educativo; por ende, es importante desarrollar competencias que conduzcan a la interpretación y a la identificación del propósito comunicativo. Así, el lector, no es como un ente pasivo: se convierte en una persona que interactúa, interroga y dialoga con el texto; llega a ser capaz de relacionar lo leído con otros conocimientos.

En el área de lenguaje es importante implementar estrategias que lleven al aprendizaje de la lectura comprensiva. Para ello se torna fundamental desarrollar en el aula una pedagogía dialógica donde se realicen acciones innovadoras que permitan al estudiante ser consciente de su propio aprendizaje mediante la autorreflexión en un contexto apropiado en el que regula su conocimiento. La importancia de saber comprender lo que se lee es fundamental a la hora de entrar en diálogo con un texto e identificar el propósito del autor.

En la compresión lectora, la macroestructura semántica se convierte en una estrategia que permite al estudiante interactuar con el texto y llegar a una lectura comprensiva. Ésta, al desarrollarse mediante el uso de organizadores gráficos, hace evidente la identificación del tópico en un discurso de forma global y coherente. 


\section{Referencias}

Bernal L., J. (1984). Tres momentos estelares en lingüistica. Bogotá: Instituto Caro y Cuervo.

Cañón, L., Mancera, J., \& Ruiz, N. (2010). La lectura de textos académicos en la formación universitaria de docentes: Entre supuestos y estrategias. Pedagogía y saberes, Revista Pedagógica Nacional, (33), 127.138. https://doi.org/10.17227 /01212494.33pys127.138

Cassany, D. (2003). Aproximaciones a la lectura crítica: teoría, ejemplos y reflexiones. Tarbiya. Revista de investigación e innovación educativa. (32).

Cerlac, OEI. (2004). Agenda de políticas públicas de lectura, Bogotá.

Frías, M. (1996). Procesos creativos para la construcción de textos. Interpretación y composición. Bogotá, Cooperativa Editorial Magisterio.

Jiménez, V. (2004). Metacognición y comprensión de la lectura: evaluación de los componentes estratégicos (procesos y variables) mediante la elaboración de una escala de conciencia lectora (escola). Memoria para optar al grado de doctor. Madrid España.

Liceo Javier de Guatemala. (2013). Lectura comprensiva y sus estrategias. Guatemala: Talleres graficos Iger.

Martínez, A., \& Rodríguez C. (1989). Sobre la didáctica del texto expositivo. Algunas propuestas para la clase de lengua. Comunicación, lenguaje y educación.

Oliveros, E. (2013) Texto expositivo: Estudio de los niveles discursivos en producciones Escritas por estudiantes de nuevo ingreso. Facultad de Humanidades. Maestría en Lingüística. República Bolivariana de Venezuela.

Rodríguez, L. (2007) Compendio de estrategias bajo el enfoque por competencias. Instituto Tecnológico de Sonora. Coordinación de desarrollo académico. Área de Innovación curricular. Primera versión. Obregón.

Rodríguez, S., Herráiz, L., Prieto, M., Martínez, M., Picazo, M., Castro, I., \& Bernal, S. (2011). Investigación Acción. Madrid, Universidad Autónoma de Madrid.

Sampieri, R., Fernández., C., \& Baptista, M. (2010). Metodología de la Investigación. Quinta Edición. México, Mc Graw Hill.

Solé, I. (1998). Estrategias de lectura. Barcelona, Graó. 
Tapia, C., Terrero, J., \& Cabral J. (2015). Metacognición, Motivación, Transferencia y Desarrollo. Santo Domingo. República Dominicana.

Van-Dijk, T. (1980) Texto y contexto. Semántica y pragmática del discurso. Madrid, Ediciones Cátedra.

Van-Dijk, T. (1996) Estructuras y funciones del discurso. México, Siglo Veintiuno Editores. 